\title{
Comparison of body water status and its distribution in patients with non- septic infection, patients with sepsis, and healthy controls
}

\author{
Jieun Shin', Inwon Park', Jae Hyuk Lee', Jong Soo Han², \\ Byunghyun Kim', Dong-Hyun Jang ${ }^{1}$, Sang-Min Lee', Che Uk Lee', \\ You Hwan Jo ${ }^{1,3}$ \\ 'Department of Emergency Medicine, Seoul National University Bundang Hospital, Seongnam, Korea
${ }^{2}$ Health Promotion Center, Seoul National University Bundang Hospital, Seongnam, Korea
${ }^{3}$ Department of Emergency Medicine, Seoul National University College of Medicine, Seoul, Korea
}

Objective Although fluid resuscitation is the cornerstone of treatment for sepsis, the role of body water status in sepsis is poorly understood. This study aimed to understand how body water and its distribution are modified in patients with sepsis and those with non-septic infection compared to healthy individuals.

Methods Two groups of adults presumed to have non-septic infection $(n=87)$ and sepsis $(n=54)$ were enrolled in this prospective study in a single emergency department, and they were compared to sex-, age-, and height-matched $(1: 3$ ratio) healthy controls $(n=11,190)$ from retrospective data in a health promotion center. Total body water (TBW), intracellular water (ICW), and extracellular water $(\mathrm{ECW})$, determined using direct segmental multi-frequent bioelectrical impedance analysis (InBody S10) were expressed as indices for normalization by body weight (BW). The ratio of ECW to TBW (ECW/TBW) was evaluated to determine body water distribution.

Results TBW/BW, ICW/BW, and ECW/BW were significantly higher in the non-septic infection group than in the healthy group $(P<0.001)$, but ECW/TBW was not significantly different $(P=0.690)$. There were no differences in TBW/BW and ICW/BW between the sepsis and healthy groups $(P=0.083$ and $P=0.963)$. However, $E C W / B W$ and $E C W / T B W$ were significantly higher in the sepsis group than in the healthy group $(P<0.001)$.

Conclusion Compared to the healthy group, the ratio of body water to BW was significantly increased in the non-septic infection group, while ECW/BW and ECW/TBW were significantly increased in the sepsis group. These indices could be utilized as diagnostic variables of body water deficit in septic patients.

Keywords Sepsis; Electric impedance; Body fluid compartments; Extracellular fluid; Intracellular fluid
elSSN: 2383-4625

Received: 27 July 2020

Revised: 26 October 2020

Accepted: 29 October 2020

Correspondence to: Inwon Park Department of Emergency Medicine, Seoul National University Bundang Hospital, 82 Gumi-ro 173beon-gil, Bundang-gu, Seongnam 13620, Korea E-mail: lifesaver@snubh.org ORCID

https://orcid.org/0000-0001-7525-9189

Jae Hyuk Lee

Department of Emergency Medicine, Seoul National University Bundang Hospital, 82 Gumi-ro 173beon-gil, Bundang-gu, Seongnam 13620, Korea E-mail: hyukmd@snubh.org ORCID https://orcid.org/0000-0003-2429-4085

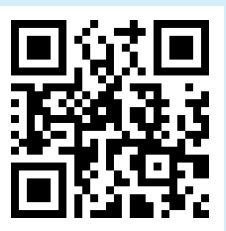

How to cite this article:

Shin J, Park I, Lee JH, Han JS, Kim B, Jang DH, Lee SM, Lee CU, Jo YH. Comparison of body water status and its distribution in patients with non-septic infection, patients with sepsis, and healthy controls. Clin Exp Emerg Med 2021;8(3):173-181. https://doi. org/10.15441/ceem.20.094

This is an Open Access article distributed under the terms of the Creative Commons Attribution Non-Commercial License (https:// creativecommons.org/licenses/by-nc/4.0/). 


Capsule
Wummat is already known
Although fluid resuscitation is critical to patients with sepsis, the body water status of patients with sepsis or non-sep-
tic infection before treatment is poorly understood which leads to subjective clinical decisions. Previous studies have
investigated the body water status in sepsis patient, nevertheless, their body water information acquired from bioelec-
trical impedance analysis is limited as a diagnostic tool due to deficiency of control data from the healthy population.
What is new in the current study
Compared to the healthy population data, the ratio of total body water to body weight was significantly increased in
patients with non-septic infection while total body water to body weight was not significantly increased in patients
with sepsis. On the other hand, the ratio of extracellular water to total body water was significantly higher in the pa-
tients with sepsis while there was no difference between non-septic infection and healthy control groups.

\section{INTRODUCTION}

The current guidelines for sepsis and septic shock treatment recommend fluid resuscitation to treat sepsis induced hypoperfusion. ${ }^{1-4}$ Although this fluid resuscitation strategy, well adopted in clinical practice, presumes dehydration in sepsis patients, pretreatment body water status in patients with sepsis has not previously been properly assessed. ${ }^{5-7}$ Moreover, recent clinical reports suggest that aggressive and indiscriminative fluid resuscitation strategies without precise identification of water status in patients are likely to worsen vasodilatory shock and increase capillary leaks and tissue edema, which can lead to an increased risk of death. ${ }^{8-10}$ Little is known about how body water percentage changes when transitioning from a healthy state to either nonseptic infection or a septic condition, and it is unclear whether patients with sepsis can be presumed to be dehydrated. ${ }^{11-13}$ Therefore, comparison of body water status in patients with non-septic infection and patients with sepsis with that in healthy individuals might help us understand the pathophysiology of the shift in body water status during the progression of sepsis. ${ }^{5,14,15}$

Using bioelectrical impedance analysis (BIA), a previous study has demonstrated that fluid resuscitation increased the proportion of extracellular water (ECW) to total body water (TBW) in a non-survivor group of patients with sepsis compared to the survivor group. ${ }^{16}$ Although the longitudinal trend of body water, including ECW/TBW, during fluid resuscitation was adequately addressed in the study, no direct comparison of body water status between patients with sepsis and healthy individuals has been conducted. It is not well understood in which water compartments body water actually alters when a previously healthy individual is diagnosed with sepsis. Due to the absence of a reference value, there has been limited use of body water status as a diagnostic parameter in patients with suspected infection including sepsis. If this alteration could be demonstrated using indices acquired from BIA, these indices would be of diagnostic value in identifying body water status as well as fluid treatment requirements in patients with sepsis.

Therefore, this study was designed to use BIA to investigate the alteration of body water status in patients with sepsis compared to healthy individuals. In addition, patients with non-septic infection were compared to the healthy population to determine how the body water profile is modified in non-septic infection and how these changes differ from those in patients with sepsis. Including patients with non-septic infection who do not require fluid resuscitation meant that diagnostic variables for fluid treatment could be evaluated.

\section{METHODS}

\section{Study settings and design}

Two groups of patients whose primary presentation to the emergency department (ED) was either sepsis or non-septic infection were enrolled prospectively from a single ED in a university affiliated hospital between January 2018 and December 2019. The third group of healthy controls who underwent routine or customized health checkups at a health promotion center in the same institute was recruited retrospectively between January 2018 and December 2018.

To prevent fluid treatment before patient enrollment, patients were enrolled after initial vital signs assessment and the first medical examination by a physician before acquiring other laboratory values or treatment. Patients in the sepsis group were those presumed to have infection with a shock feature (defined as systolic blood pressure $\leq 90 \mathrm{mmHg}$ ) necessitating fluid therapy. Patients 
in the non-septic infection group were those with probable infection according to initial vital signs and first medical examination but who did not show shock or organ failure features requiring fluid treatment. ${ }^{17}$ These two groups of patients were then compared to sex-, age- ( \pm 1 year $)$, and height- $( \pm 2 \mathrm{~cm})$ matched healthy controls in a 1:3 ratio. ${ }^{18,19}$ Patients who were aged $<18$ years, had end-stage renal disease with renal replacement therapy, presented with overt heart failure, had a pacemaker or an implantable cardioverter defibrillator, or had received any fluid therapy intravenously prior to enrollment were excluded from the sepsis and non-septic infection groups. Patients transferred from lower level hospitals were not excluded on that basis as long as they did not have intravenous fluid therapy prior to ED arrival. Prior fluid administration was confirmed by either the patient or the guardian. Written informed consent was obtained from all patients or their legal guardians before enrollment in the study. Retrospective data acquisition from healthy controls was approved by the health promotion center. All study procedures were performed in accordance with the relevant guidelines and regulations. The study was approved by the institutional review board of Seoul National University Bundang Hospital (B-1911/577-305).

\section{Measurement of clinical variables}

Data related to baseline demographics, including age, sex, and underlying diseases, such as diabetes mellitus, hypertension, chronic liver disease, chronic lung disease, and chronic kidney disease, were collected for patient and healthy groups. Height $(\mathrm{cm})$ and weight $(\mathrm{kg})$ data required in BIA measurements were acquired before fluid treatment for the non-septic and sepsis patient groups. If weight measurement was not feasible due to the patient's bedridden status or very unstable vital signs, data were acquired from previous electronic medical records or information from either the patient or the guardian. For patients in the sepsis and nonseptic infection groups, initial vital signs, including systolic blood pressure, diastolic blood pressure, mean arterial pressure, heart rate, respiratory rate, and body temperature, were measured. Source of infection was assessed by retrospectively reviewing medical records or based on discharge diagnosis provided by the attending physician. Laboratory values, including complete blood counts and total bilirubin, blood urea nitrogen, creatinine, albumin, sodium, potassium, calcium, phosphorus, glucose, uric acid, and C-reactive protein levels, were obtained from electronic medical records.

\section{BIA}

InBody S10 and 770 (InBody Co., Seoul, Korea) devices were used to analyze body water status in patients recruited in the ED and healthy controls, respectively. Eight electrodes, two electrodes for each of the four extremities, were attached to the individuals in the supine position. Thirty impedance variables using six different frequencies $(1,5,50,250,500$, and $1,000 \mathrm{kHz})$ at each of the five parts of the body (the right arm, left arm, trunk, right leg, and left

Table 1. Baseline characteristics of study population

\begin{tabular}{|c|c|c|c|c|}
\hline Variable & $\begin{array}{c}\text { Healthy } \\
\text { volunteers } \\
(n=11,190)\end{array}$ & $\begin{array}{l}\text { Non-septic } \\
\text { infections } \\
(n=87)\end{array}$ & $\begin{array}{c}\text { Sepsis } \\
\text { patients } \\
(n=54)\end{array}$ & P-value \\
\hline Age & $49.6 \pm 11.8$ & $59.6 \pm 17.6$ & $72.9 \pm 10.9$ & $<0.001$ \\
\hline Sex, male & 6,354 (56.8) & $45(51.7)$ & $35(64.8)$ & 0.312 \\
\hline Height (cm) & $166.7 \pm 8.4$ & $163.2 \pm 9.1$ & $160.9 \pm 9.6$ & $<0.001$ \\
\hline Weight (kg) & $67.0 \pm 12.9$ & $61.5 \pm 12.8$ & $53.3 \pm 11.5$ & $<0.001$ \\
\hline \multicolumn{5}{|l|}{ Underlying disease } \\
\hline Hypertension & $2,906(26.0)$ & $27(31.0)$ & $25(46.3)$ & 0.002 \\
\hline Diabetes mellitus & $1,141(10.2)$ & $17(19.5)$ & $19(35.2)$ & $<0.001$ \\
\hline Chronic liver disease & $179(1.6)$ & $6(6.9)$ & $6(11.1)$ & $<0.001$ \\
\hline Chronic lung disease & $310(2.8)$ & $8(9.2)$ & $8(14.8)$ & $<0.001$ \\
\hline Chronic kidney disease & $63(0.6)$ & $3(3.4)$ & $4(7.4)$ & $<0.001$ \\
\hline \multicolumn{5}{|l|}{ Initial vital sign } \\
\hline $\mathrm{SBP}(\mathrm{mmHg})$ & NA & $127.2 \pm 20.2$ & $81.2 \pm 11.6$ & $<0.001$ \\
\hline $\mathrm{DBP}(\mathrm{mmHg})$ & NA & $71.5 \pm 11.9$ & $49.2 \pm 11.4$ & $<0.001$ \\
\hline $\operatorname{MAP}(\mathrm{mmHg})$ & NA & $90.1 \pm 13.1$ & $59.9 \pm 9.9$ & $<0.001$ \\
\hline HR (bpm) & NA & $101.4 \pm 18.0$ & $97.3 \pm 23.9$ & 0.240 \\
\hline RR (rate) & NA & $19.8 \pm 4.6$ & $23.2 \pm 12.4$ & 0.021 \\
\hline $\mathrm{BT}\left({ }^{\circ} \mathrm{C}\right)$ & NA & $38.0 \pm 0.9$ & $36.9 \pm 2.7$ & $<0.001$ \\
\hline Source of infection & & & & 0.688 \\
\hline Respiratory & NA & $57(65.5)$ & $31(57.4)$ & \\
\hline Hepatobiliary & NA & $13(14.9)$ & $10(18.5)$ & \\
\hline Gastrointestinal & NA & $5(5.8)$ & $3(5.6)$ & \\
\hline Genitourinary & NA & 8 (9.2) & $9(16.7)$ & \\
\hline Musculoskeletal & NA & $3(3.5)$ & $1(1.9)$ & \\
\hline Neurological & NA & $1(1.2)$ & $0(0.0)$ & \\
\hline \multicolumn{5}{|l|}{ Laboratory findings } \\
\hline White blood cells $\left(\times 10^{3} / \mu \mathrm{L}\right)$ & NA & $9.9 \pm 5.1$ & $11.7 \pm 8.0$ & 0.100 \\
\hline Hemoglobin (g/dL) & NA & $12.7 \pm 2.1$ & $10.7 \pm 2.0$ & $<0.001$ \\
\hline Platelets $\left(\times 10^{3} / \mu \mathrm{L}\right)$ & NA & $228.4 \pm 108.2$ & $157.9 \pm 106.4$ & $<0.001$ \\
\hline Total bilirubin (mg/dL) & NA & $1.1 \pm 1.4$ & $2.5 \pm 4.9$ & 0.019 \\
\hline Blood urea nitrogen $(\mathrm{mg} / \mathrm{dL})$ & NA & $18.9 \pm 21.6$ & $38.5 \pm 28.7$ & $<0.001$ \\
\hline Creatinine $(\mathrm{mg} / \mathrm{dL})$ & NA & $1.0 \pm 1.8$ & $1.7 \pm 1.1$ & 0.020 \\
\hline Albumin $(\mathrm{g} / \mathrm{dL})$ & NA & $3.7 \pm 0.5$ & $2.9 \pm 0.6$ & $<0.001$ \\
\hline Sodium (mmol/L) & NA & $136.9 \pm 4.1$ & $137.2 \pm 9.3$ & 0.760 \\
\hline Potassium (mmol/L) & NA & $4.2 \pm 0.5$ & $4.3 \pm 0.8$ & 0.180 \\
\hline Calcium (mg/dL) & NA & $8.7 \pm 0.8$ & $8.3 \pm 0.8$ & $<0.001$ \\
\hline Phosphorus (mg/dL) & NA & $3.4 \pm 1.4$ & $3.8 \pm 1.7$ & 0.110 \\
\hline Glucose (mg/dL) & NA & $127.7 \pm 43.7$ & $146.4 \pm 103.0$ & 0.140 \\
\hline Uric acid (mg/dL) & NA & $4.6 \pm 1.9$ & $6.3 \pm 3.4$ & $<0.001$ \\
\hline C-reactive protein (mg/dL) & NA & $7.7 \pm 6.7$ & $14.2 \pm 8.8$ & $<0.001$ \\
\hline
\end{tabular}

Values are presented as mean \pm standard deviation or number (\%).

SBP, systolic blood pressure; NA, not applicable; DBP, diastolic blood pressure; $M A P$, mean arterial pressure; $H R$, heart rate; $R R$, respiratory rate; $B T$, body temperature. 
leg) and 15 reactance $\left(X_{c}\right)$ and phase angle $(\theta)$ variables using three different frequencies $(5,50$, and $250 \mathrm{kHz})$ at each of the five parts of the body were measured. Phase angle, a well-known parameter in previous BIA studies, was also investigated to compare with other variables. ${ }^{20-22} \mathrm{TBW}$, intracellular water (ICW), and ECW were estimated using the differences in electrical conductance measured by the devices. All BIA measurements were performed before fluid resuscitation to reflect the pretreatment body water status.

\section{Statistical analysis}

For sample size determination, the matching of $1: n$ ratio of patients to controls were conducted; although a healthy population of 11,190 were recruited, only up to $1: 3$ ratio of patients to controls was feasible due to the extremely old population in the group of sepsis patients. Continuous variables are presented as mean \pm standard deviation and categorical variables are presented as numbers (\%). Analysis of variance with a post-hoc Tukey multiple comparisons test was conducted to determine the differences between the healthy, non-septic, and sepsis groups. Student t-test was
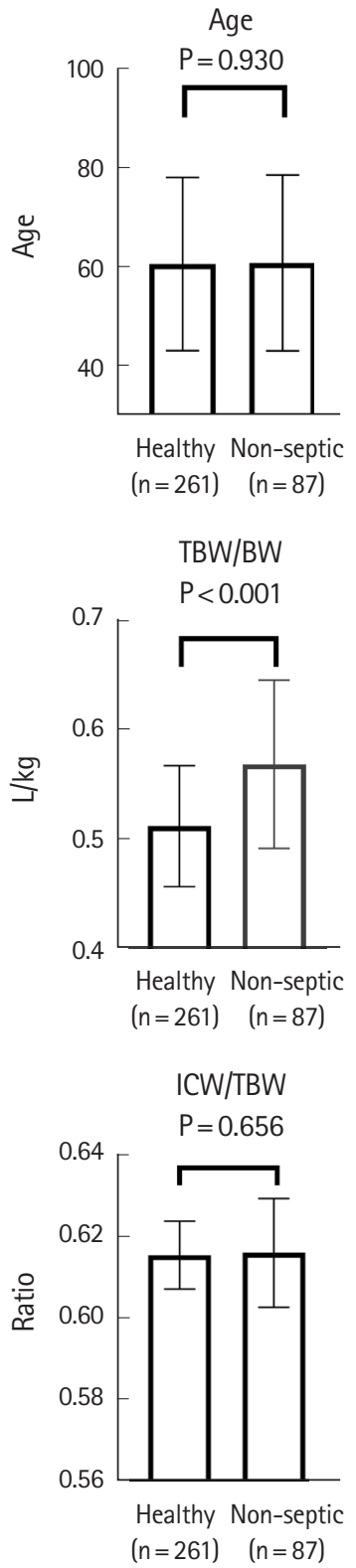
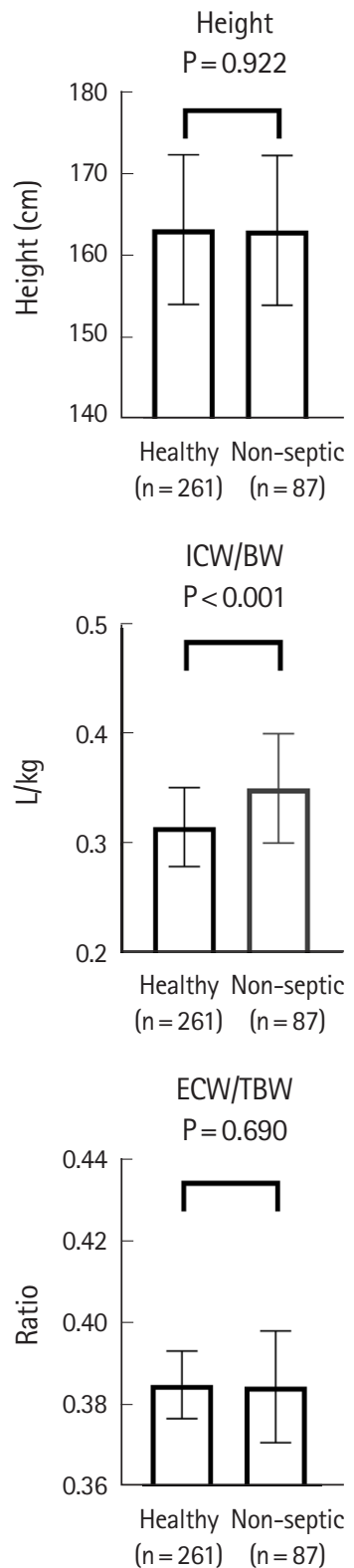
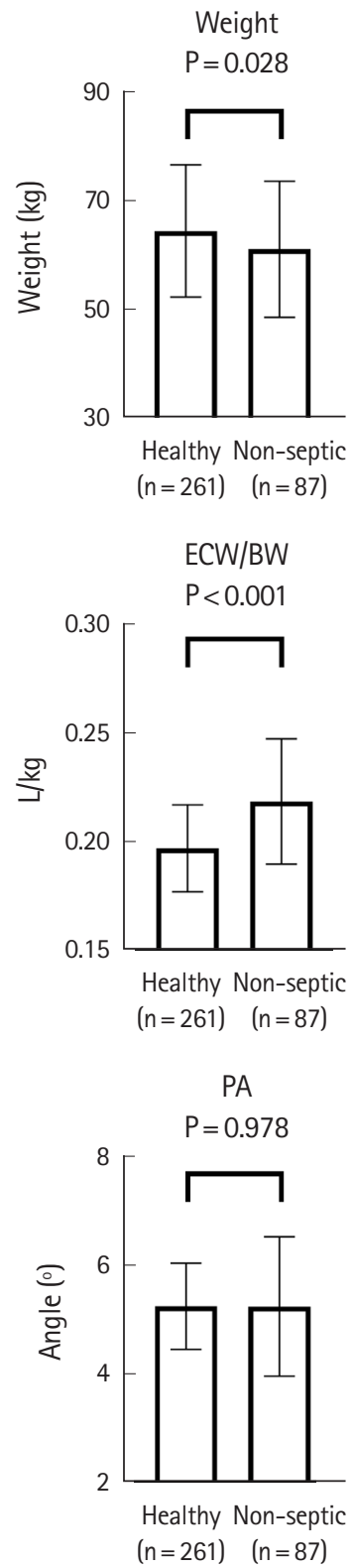

Fig. 1. Comparison of age, height, weight, bioelectrical impedance analysis indices, and phase angle variables in matched groups of healthy and nonseptic individuals. Data are presented as the mean \pm standard deviation. $n$ refers to the size of the sample group. TBW, total body water; BW, body weight; ICW, intracellular water; ECW, extracellular water; PA, phase angle. 
used to determine P-values, and $\mathrm{P}<0.05$ was considered statistically significant. Statistical analyses were conducted using STATA/ SE ver. 15.0 (StataCorp LP, College Station, TX, USA) and Prism ver. 8.0 (GraphPad Software, San Diego, CA, USA).

\section{RESULTS}

Study population and baseline characteristics

Eighty-seven patients with non-septic infection and 54 patients with sepsis were prospectively enrolled during the study period.
The retrospectively recruited control population included 11,190 healthy individuals. The baseline characteristics of all three groups are shown in Table 1. Age, height, and weight were significantly different across the three groups; more details of these differences and their significance are shown in Supplementary Fig. 1. The proportions of underlying diseases also varied significantly among the groups, with the sepsis group having the highest proportion of underlying co-morbidities. Initial vital signs were also significantly different between the non-septic infection and sepsis groups, whereas the sites of infection were distributed in a similar pat-
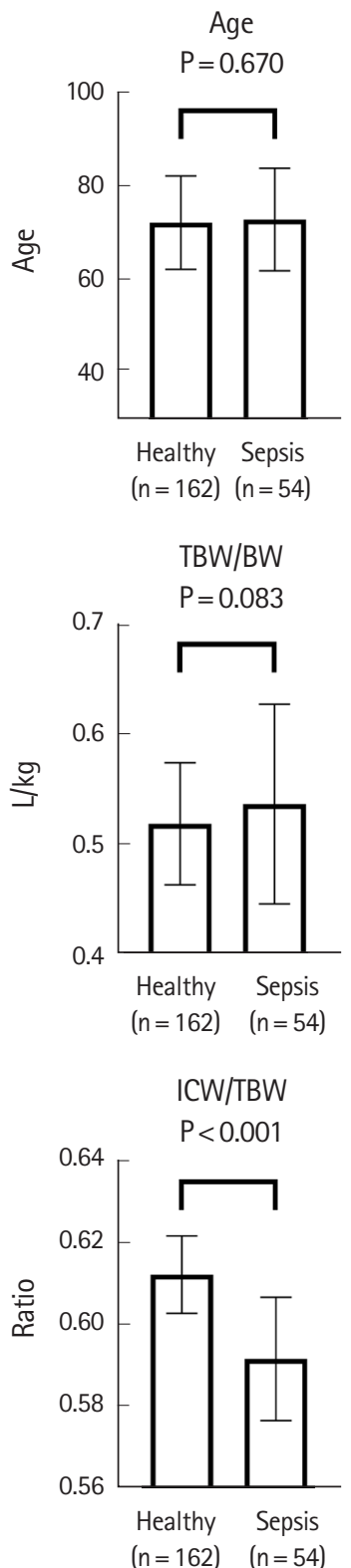
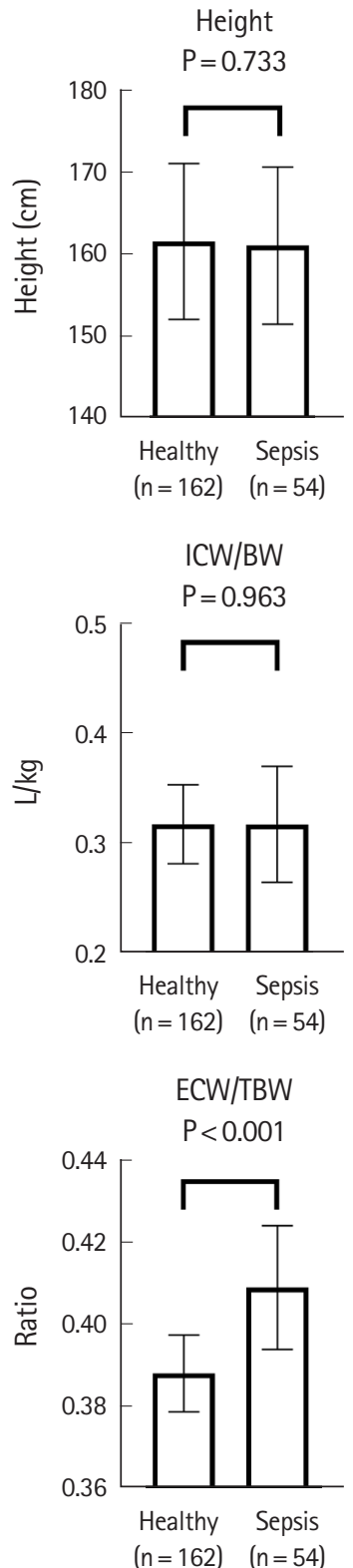
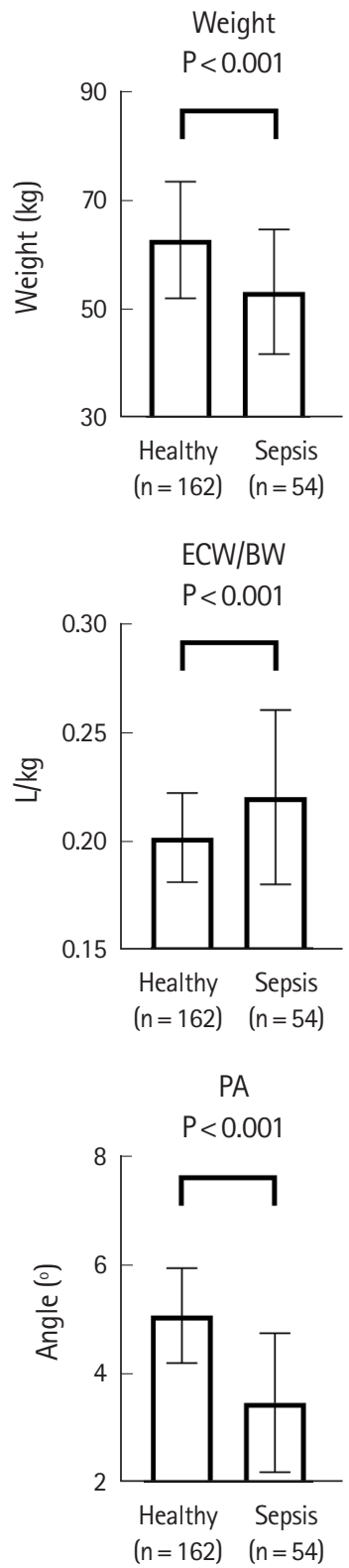

Fig. 2. Comparison of age, height, weight, bioelectrical impedance analysis indices, and phase angle variables in matched groups of healthy and sepsis individuals. Data are presented as the mean \pm standard deviation. $n$ refers to the size of the sample group. TBW, total body water; BW, body weight; ICW, intracellular water; ECW, extracellular water; PA, phase angle. 
tern in both groups of patients. Among laboratory findings, white blood cell counts did not vary significantly, whereas hemoglobin and platelet counts were significantly decreased in the sepsis group. Total bilirubin, blood urea nitrogen, creatinine, uric acid, and Creactive protein were significantly higher in the sepsis group, while albumin and calcium levels were significantly lower.

\section{Body water status in patients with sepsis and those with non-septic infection compared to healthy controls}

The TBW, ICW, and ECW values obtained from BIA were standardized by body weight (BW) and expressed as TBW/BW, ICW/BW, and ECW/BW. In addition, the ratio of ICW to TBW (ICW/TBW) and the ratio of ECW to TBW (ECW/TBW) were calculated to compare the distribution of body water. Apart from body water profiles, phase angles, which were automatically calculated by the BIA device and reflected the ratio of body cell mass to fat free mass, were compared (Supplementary Fig. 1). The resulting bar plots show distinct differences between the proportions of indices obtained from BIA. To control for confounding factors in baseline characteristics, patients with non-septic infection and healthy controls (Fig. 1) and patients with sepsis and healthy controls (Fig. 2) were sex-, age- $( \pm 1$ year), and height-matched $( \pm 2 \mathrm{~cm})$ in a 1:3 ratio.

The resulting data from the matching process are shown in Figs. 1 and 2. TBW/BW $(P<0.001)$, ICW/BW $(P<0.001)$, and ECW/ BW $(P<0.001)$ were significantly increased in the non-septic infection group compared with the healthy group; however, there were no significant differences in ICW/TBW or ECW/TBW and phase angles.

Fig. 2 shows the differences in values between the sepsis and healthy groups. Unlike when comparing the non-septic infection and healthy groups, TBW/BW and ICW/BW were not significantly decreased in the sepsis group compared with the healthy group, while the ECW/TBW increased significantly $(P<0.001)$ in the sepsis group. ICW/TBW $(P<0.001)$ and phase angles $(P<0.001)$ were also significantly lower in the sepsis group.

\section{DISCUSSION}

This study demonstrated body water profile using TBW, ICW, and ECW values obtained using BIA. The distribution of body water in patients with non-septic infection and those with sepsis was significantly different from healthy controls. To exclude confounding factors, such as sex, age, and height, retrospective control data from a health promotion center were used, and three healthy controls were sex-, age-, and height-matched to each patient diagnosed with non-septic infection or sepsis. ${ }^{18,19}$ Compared to the healthy population, TBW, including ECW and ICW, was significantly increased in patients with non-septic infection, while TBW was not decreased in patients with sepsis. In contrast, the ECW/ TBW, which indicates extracellular edema and intracellular dehydration, was significantly higher in patients with sepsis, whereas no difference was identified between the non-septic infection and healthy groups.

BIA is of interest as a clinical tool in the assessment of volume status because it is an inexpensive, quick, and non-invasive measuring tool, as well as an accessible one. ${ }^{23-25}$ BIA allows for vector analysis, phase angle derivation, and direct segmental multi-frequency BIA specifically measures TBW, as well as ICW and ECW water content, which provides insight into the destination of administered fluids. ${ }^{26-28}$ However, despite the increasing popularity of BIA in clinical practice, previous studies of BIA in sepsis patients provide only limited evidence for the variable having diagnostic value, because of the lack of comparisons with the healthy population. As BIA measurements are strongly influenced by sex, age, and height, comparisons might be acceptable if these factors were matched. ${ }^{29}$ One strength of our study is that these matching comparisons were performed, thus minimizing confounding bias. In addition, as we have previously demonstrated, fluid treatment alters the body water status, which suggests that measurement of BIA could be conducted before the initiation of fluid administration to accurately calculate body water status in an unbiased manner. 16,23

Previous studies have assessed the feasibility and analyzed the volume status of sepsis patients using BIA. In a single intensive care unit study, patients with peritonitis and sepsis had higher ECW components than those with blunt trauma, although changes in TBW were similar. ${ }^{12}$ In another study using continuous renal replacement therapy to correct volume excess in septic shock patients with acute renal failure, there was an increase in TBW, ECW, and ECW/ICW in non-survivors. ${ }^{30} \mathrm{~A}$ third study comparing intensive care unit patients with sepsis to those without sepsis showed that ECW and fat free mass hydration were increased in severe sepsis compared to sepsis. ${ }^{31}$ However, even though the increase in ECW was similar to our study's finding, the above-mentioned studies were limited in their ability to suggest that ECW variables have initial diagnostic value, because direct comparisons with healthy volunteers were absent.

Our results, showing a significant increase in ECW/TBW ratio but no significant increase in TBW/BW ratio in the sepsis group may be explained by the hypothesis that a systemic inflammatory response with oxidative stress and production of reactive oxygen species is associated with damage to cell membranes and loss of cell wall integrity that results in capillary leakage and interstitial 
edema. ${ }^{32-34}$ In addition, patients with sepsis are prone to the distribution of fluid from the intravascular to the extravascular space, which does not respond to the usual massive fluid treatment strategy. ${ }^{35}$ Therefore, we suggest that the ECW/TBW ratio may in the future be used as a diagnostic tool to assess sepsis-related capillary leakage syndrome. ${ }^{16,36}$ An increasing ECW/TBW can be a detrimental sign for fluid treatment in sepsis, and based on this hypothesis we are currently planning a further clinical trial investigating the ECW/TBW index as a monitoring parameter for fluid treatment. ${ }^{16}$ Besides this, additional future studies are necessary to investigate ways of improving cellular and vascular integrity. ${ }^{33,37}$

Phase angle is a significant predictor of survival in not only sepsis but also various other diseases, according to previous BIA studies. ${ }^{38-40}$ It is regarded as a biomarker of cellular health, as it is correlated with cell mass, the integrity of cell membranes, and hydration profile. ${ }^{24,41}$ In our study, a significant difference in phase angle was also identified between the healthy controls and patients with sepsis, as well as the significant difference in ECW/ TBW. However, as shown in the previous study, a longitudinal trend of phase angle failed to differentiate between survivors and nonsurvivors of sepsis, thus its value as a monitoring marker in fluid treatment is limited. ${ }^{16}$

Interestingly, compared to the matched healthy subjects, patients with non-septic infection presented with significantly increased TBW/BW, ICW/BW, and ECW/BW. In contrast, the proportions of ICW and ECW to TBW were not significantly different, suggesting fluid retention without an alteration of body water distribution in patients with non-septic infection. The reason for this phenomenon is not completely understood and a possible explanation could be that in certain acute infections, pro-inflammatory cytokines induced by inflammation increase the release of arginine vasopressin in the posterior pituitary gland. ${ }^{42}$ Further research addressing water retention caused by antidiuretic hormone elevation in infectious diseases may be warranted. ${ }^{43-45}$

This study has several limitations. First, the study was limited to a small number of patients and healthy subjects of a single ethnicity and in a single tertiary center. Future multicenter studies or studies including individuals of other ethnicities might be needed for greater generalizability. Second, we could match and compare the healthy group with the non-septic infection group and the healthy group with the sepsis group, but we could not directly compare the non-septic infection and sepsis groups because of the significantly different age ranges of individuals in those two groups (Table 1). Third, we could not control each person's intake of water before enrollment into the study due to the characteristics of the study. Bias from the water deprivation of healthy controls, due to work-ups at the health promotion center, or poor oral intake of sepsis patients cannot be fully excluded. We did, however, conduct a chart review of healthy controls to exclude those who had a bowel preparation before colonoscopy. Fourth, although we excluded patients with an underlying volume overload condition such as heart failure or with end-stage renal disease to minimize the possible influence on the body water measurements, we did not include other conditions such as lymphedema in our exclusion criteria. Fortunately, on retrospective review, we found that none of the included patients in either the sepsis or non-septic infection group was previously diagnosed with lymphedema. Fifth, although previous studies have utilized direct segmental multi-frequency BIA of a specific instrument (InBody S10 and InBody 770), BIA is not fully validated for body water measurement in patients with sepsis. ${ }^{16,38,46}$ As BIA might not be the gold standard for determining body water, further external validation in different clinical conditions or direct comparison with the water dilution method might be needed. ${ }^{12}$ Finally, measurement of BW, which was utilized as a standardization variable for body water, was not feasible in some patients with sepsis due to their very unstable clinical condition or requirement of inbed measurements in crowded ED situations. In those patients, or in cases where guardians could not accurately remember the BW, $B W$ variables might have been inaccurate.

In summary, compared to the healthy group, the ratio of body water to BW was significantly increased in the non-septic infection group, while ECW/BW and ECW/TBW were significantly increased in the sepsis group. Instead of body water decrease, modification of body water distribution to the extracellular portion might be a potential diagnostic variable to body water deficit in patients with sepsis.

\section{CONFLICT OF INTEREST}

No potential conflict of interest relevant to this article was reported.

\section{ACKNOWLEDGMENTS}

The authors thank Ji Eun Lee and Ji Woo Lee for their assistance in patient enrollment.

\section{SUPPLEMENTARY MATERIAL}

Supplementary Fig is available from: https://doi.org/10.15441/ ceem.20.094. 


\section{REFERENCES}

1. Rhodes A, Evans LE, Alhazzani W, et al. Surviving Sepsis Campaign: international guidelines for management of sepsis and septic shock. 2016. Crit Care Med 2017;45:486-552.

2. ProCESS Investigators, Yealy DM, Kellum JA, et al. A randomized trial of protocol-based care for early septic shock. N Engl J Med 2014;370:1683-93.

3. ARISE Investigators; ANZICS Clinical Trials Group, Peake SL, et al. Goal-directed resuscitation for patients with early septic shock. N Engl J Med 2014;371:1496-506.

4. Levy MM, Evans LE, Rhodes A. The Surviving Sepsis Campaign Bundle: 2018 update. Intensive Care Med 2018;44:925-8.

5. Marik P, Bellomo R. A rational approach to fluid therapy in sepsis. Br J Anaesth 2016;116:339-49.

6. RENAL Replacement Therapy Study Investigators, Bellomo R, Cass $A$, et al. An observational study fluid balance and patient outcomes in the Randomized Evaluation of Normal vs. Augmented Level of Replacement Therapy trial. Crit Care Med 2012;40:1753-60.

7. Finn PJ, Plank LD, Clark MA, Connolly AB, Hill GL. Progressive cellular dehydration and proteolysis in critically ill patients. Lancet 1996;347:654-6.

8. Kelm DJ, Perrin JT, Cartin-Ceba R, Gajic 0, Schenck L, Kennedy CC. Fluid overload in patients with severe sepsis and septic shock treated with early goal-directed therapy is associated with increased acute need for fluid-related medical interventions and hospital death. Shock 2015;43:68-73.

9. Maitland K, Kiguli S, Opoka RO, et al. Mortality after fluid bolus in African children with severe infection. $N$ Engl J Med 2011;364:2483-95.

10. Tigabu BM, Davari M, Kebriaeezadeh A, Mojtahedzadeh M. Fluid volume, fluid balance and patient outcome in severe sepsis and septic shock: a systematic review. J Crit Care 2018; 48:153-9.

11. Murugan $R$, Kellum JA. Fluid balance and outcome in acute kidney injury: is fluid really the best medicine? Crit Care Med 2012;40:1970-2.

12. Plank LD, Hill GL. Similarity of changes in body composition in intensive care patients following severe sepsis or major blunt injury. Ann N Y Acad Sci 2000;904:592-602.

13. Schwenk A, Schlottmann S, Kremer G, Diehl V, Salzberger B, Ward L. Fever and sepsis during neutropenia are associated with expansion of extracellular and loss of intracellular water. Clin Nutr 2000;19:35-41.

14. Brown RM, Semler MW. Fluid management in sepsis. J Intensive Care Med 2019;34:364-73.
15. Marx G. Fluid therapy in sepsis with capillary leakage. Eur J Anaesthesiol 2003;20:429-42.

16. Park I, Lee JH, Jang DH, et al. Assessment of body water distribution in patients with sepsis during fluid resuscitation using multi-frequency direct segmental bioelectrical impedance analysis. Clin Nutr 2020;39:1826-31.

17. Singer M, Deutschman CS, Seymour CW, et al. The third international consensus definitions for sepsis and septic shock (Sepsis-3). JAMA 2016;315:801-10.

18. Kyle UG, Genton L, Pichard C. Low phase angle determined by bioelectrical impedance analysis is associated with malnutrition and nutritional risk at hospital admission. Clin Nutr 2013; 32:294-9.

19. Kyle UG, Pirlich M, Lochs H, Schuetz T, Pichard C. Increased length of hospital stay in underweight and overweight patients at hospital admission: a controlled population study. Clin Nutr 2005;24:133-42.

20. Berbigier MC, Pasinato VF, Rubin Bde A, Moraes RB, Perry ID. Bioelectrical impedance phase angle in septic patients admitted to intensive care units. Rev Bras Ter Intensiva 2013;25:2531.

21. Colin-Ramirez E, Castillo-Martinez L, Orea-Tejeda A, VazquezDuran M, Rodriguez AE, Keirns-Davis C. Bioelectrical impedance phase angle as a prognostic marker in chronic heart failure. Nutrition 2012;28:901-5.

22. Selberg 0 , Selberg D. Norms and correlates of bioimpedance phase angle in healthy human subjects, hospitalized patients, and patients with liver cirrhosis. Eur J Appl Physiol 2002;86: 509-16.

23. Kyle UG, Bosaeus I, De Lorenzo AD, et al. Bioelectrical impedance analysis: part I. Review of principles and methods. Clin Nutr 2004;23:1226-43.

24. Kyle UG, Bosaeus I, De Lorenzo AD, et al. Bioelectrical impedance analysis: part II. Utilization in clinical practice. Clin Nutr 2004;23:1430-53.

25. Bedogni G, Malavolti M, Severi S, et al. Accuracy of an eightpoint tactile-electrode impedance method in the assessment of total body water. Eur J Clin Nutr 2002;56:1143-8.

26. Gibson AL, Holmes JC, Desautels RL, Edmonds LB, Nuudi L. Ability of new octapolar bioimpedance spectroscopy analyzers to predict 4-component-model percentage body fat in Hispanic, black, and white adults. Am J Clin Nutr 2008;87: 332-8.

27. Ling $\mathrm{CH}_{\text {, de }}$ Craen $\mathrm{A}$, Slagboom PE, et al. Accuracy of direct segmental multi-frequency bioimpedance analysis in the assessment of total body and segmental body composition in middle-aged adult population. Clin Nutr 2011;30:610-5. 
28. Sartorio A, Malavolti M, Agosti F, et al. Body water distribution in severe obesity and its assessment from eight-polar bioelectrical impedance analysis. Eur J Clin Nutr 2005;59:15560.

29. Barbosa-Silva MC, Barros AJ, Wang J, Heymsfield SB, Pierson RN Jr. Bioelectrical impedance analysis: population reference values for phase angle by age and sex. Am J Clin Nutr 2005; 82:49-52.

30. Dabrowski W, Kotlinska-Hasiec E, Schneditz D, et al. Continuous veno-venous hemofiltration to adjust fluid volume excess in septic shock patients reduces intra-abdominal pressure. Clin Nephrol 2014;82:41-50.

31. Malbrain ML, Huygh J, Dabrowski W, De Waele JJ, Staelens A, Wauters J. The use of bio-electrical impedance analysis (BIA) to guide fluid management, resuscitation and deresuscitation in critically ill patients: a bench-to-bedside review. Anaesthesiol Intensive Ther 2014;46:381-91.

32. Goldenberg NM, Steinberg BE, Slutsky AS, Lee WL. Broken barriers: a new take on sepsis pathogenesis. Sci Transl Med 2011;3:88ps25.

33. Angus DC, van der Poll T. Severe sepsis and septic shock. $N$ Engl J Med 2013;369:840-51.

34. Han S, Lee SJ, Kim KE, et al. Amelioration of sepsis by TIE2 activation-induced vascular protection. Sci Transl Med 2016; 8:335ra55.

35. Malbrain MLNG, Van Regenmortel N, Saugel B, et al. Principles of fluid management and stewardship in septic shock: it is time to consider the four D's and the four phases of fluid therapy. Ann Intensive Care 2018;8:66.

36. Marx G, Vangerow B, Burczyk $C$, et al. Evaluation of noninvasive determinants for capillary leakage syndrome in septic shock patients. Intensive Care Med 2000;26:1252-8.

37. Fink MP, Warren HS. Strategies to improve drug development for sepsis. Nat Rev Drug Discov 2014;13:741-58.
38. Lee YH, Lee JD, Kang DR, Hong J, Lee JM. Bioelectrical impedance analysis values as markers to predict severity in critically ill patients. J Crit Care 2017;40:103-7.

39. Azevedo ZM, Santos Junior BD, Ramos EG, et al. The importance of bioelectrical impedance in the critical pediatric patient. Clin Nutr 2020;39:1188-94.

40. Stapel SN, Looijaard WGPM, Dekker IM, Girbes ARJ, Weijs PJM, Oudemans-van Straaten HM. Bioelectrical impedance analysis-derived phase angle at admission as a predictor of 90-day mortality in intensive care patients. Eur J Clin Nutr 2018;72:1019-25.

41. Lukaski HC, Kyle UG, Kondrup J. Assessment of adult malnutrition and prognosis with bioelectrical impedance analysis: phase angle and impedance ratio. Curr Opin Clin Nutr Metab Care 2017;20:330-9.

42. Swart RM, Hoorn EJ, Betjes MG, Zietse R. Hyponatremia and inflammation: the emerging role of interleukin-6 in osmoregulation. Nephron Physiol 2011;118:45-51.

43. Lee LC, Noronha M. When plenty is too much: water intoxication in a patient with a simple urinary tract infection. BMJ Case Rep 2016;2016:bcr2016216882.

44. Sharples PM, Seckl JR, Human D, Lightman SL, Dunger DB. Plasma and cerebrospinal fluid arginine vasopressin in patients with and without fever. Arch Dis Child 1992;67:9981002.

45. van Steensel-Moll HA, Hazelzet JA, van der Voort E, Neijens $\mathrm{HJ}$, Hackeng WH. Excessive secretion of antidiuretic hormone in infections with respiratory syncytial virus. Arch Dis Child 1990;65:1237-9.

46. Kim D, Sun JS, Lee YH, Lee JH, Hong J, Lee JM. Comparative assessment of skeletal muscle mass using computerized tomography and bioelectrical impedance analysis in critically ill patients. Clin Nutr 2019;38:2747-55. 


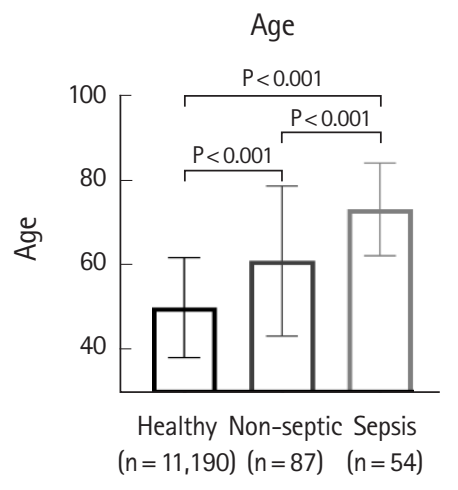

TBW/BW

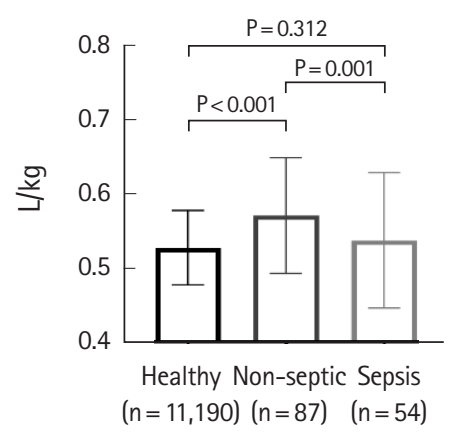

ICW/TBW

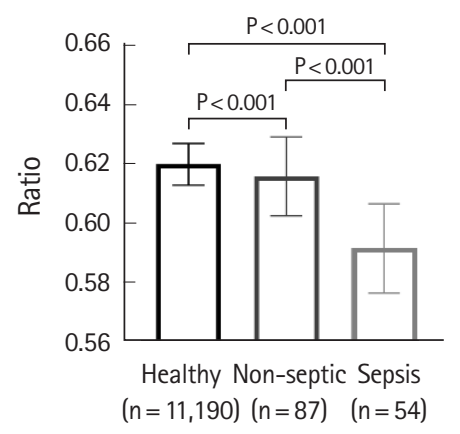

Height

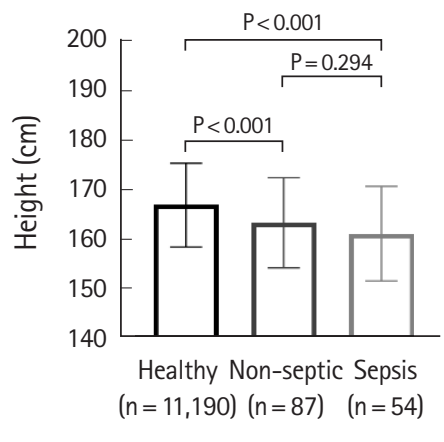

ICW/BW

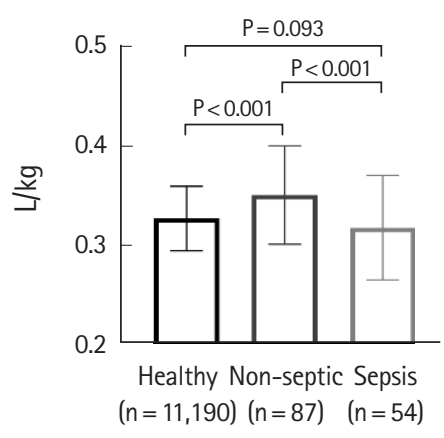

ECW/TBW

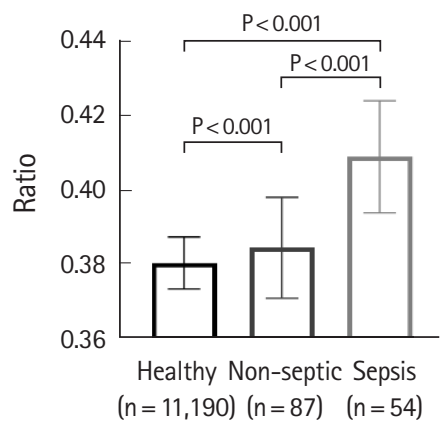

Weight

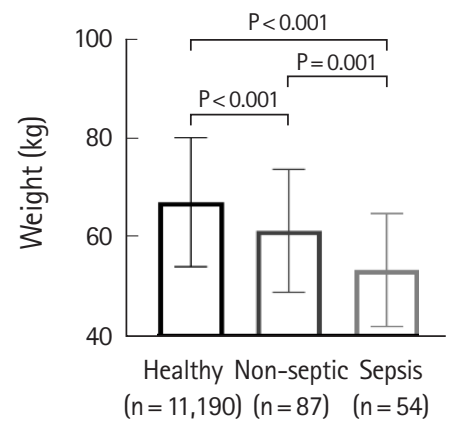

\section{ECW/BW}

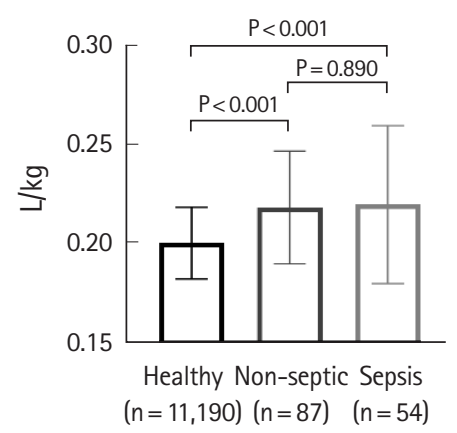

Phase angle

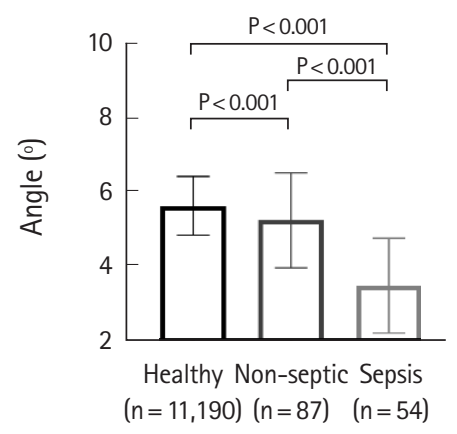

Supplementary Fig. 1. Comparison of age, height, weight, TBW/BW, ICW/BW, ECW/BW, ICW/TBW, ECW/TBW, and phase angle variables in raw groups of healthy, non-septic, sepsis. Data are presented as the mean \pm standard deviation. $n$ refers to the number of each group and P-values of the post-hoc comparison tests are shown. TBW, total body water; BW, body weight; ICW, intracellular water; ECW, extracellular water. 